\title{
New method for generation of short high-power rf pulses
}

\author{
M. A. Dorf and A.V. Savilov* \\ Institute of Applied Physics, Russian Academy of Sciences, 46 Ulyanov Street, Nizhny Novgorod, 603950, Russian Federation
}

(Received 9 September 2004; published 22 November 2004)

\begin{abstract}
The effect of "pressing out" of a short rf powerful pulse by an electron beam from a Bragg cavity pumped by a low-power input rf signal is studied. This effect occurs as a result of the transient process from the "cold" (without the electron beam) steady state of the system to the "hot" one. It is shown that the peak power of the output pulse can be significantly higher than both the pumping rf power and the electron beam power. As for the duration of the output pulse, it is fixed by the cold characteristics of the microwave system.
\end{abstract}

DOI: 10.1103/PhysRevSTAB.7.112001

PACS numbers: 41.60.Cr, 42.25.Fx

\section{INTRODUCTION}

Various methods of generating short broadband powerful rf pulses have been subjects of recent investigations. One could mention rf pulse compressors [1-4], electron generators based on various types of super-radiation of short electron bunches [5,6], as well as electron autooscillators (mainly backward-wave tubes), where a powerful short output rf pulse is formed as a result of a complicated transient spatiotemporal process of excitation of the oscillator in the case when the electron current exceeds significantly the starting level [7,8].

In this work we propose an original method for generation of the short powerful rf pulses. The method is based on "pressing out" of an rf pulse from a Bragg cavity (see, e.g., [9-11]), which is pumped with a long lowpower input signal, by the front of a short electron pulse (Fig. 1). The eigenmode of the "cold" (without the electron beam) cavity is formed by two traveling counterpropagating waves, which are coupled on the Bragg structure [Fig. 1(a)]. After the cold steady state builds up, a beam of electron oscillators enters the cavity [Fig. 1(b)]. Because of the resonant interaction between electrons and the forward (copropagating) component of the cavity eigenmode, the electron beam perturbs the cold mode slightly. Thus, the electron beam initiates a transient process from the cold steady state to the "hot" one. It is important that in the beginning of this transient process a short high-power rf pulse can be radiated from the cavity output [Fig. 1(c)].

It is important that, in contrast to active rf pulse compressors [1-4], the hot eigen mode just slightly differs from the cold one, so that the energy of the output pulse is much lower than the rf energy stored inside the cavity. Nevertheless, at some conditions the peak output pulse power can be as high as the power of the traveling $\mathrm{rf}$ waves circulating across the cavity, and it is much higher

\footnotetext{
*Phone number: +7-8312-164818.

Fax number: +7-8312-160616.

Email address: savilov@appl.sci-nnov.ru
}

than both the power of the input rf signal and the electron beam power. It is important also that the duration of the output rf pulse is fixed only by the cold parameters of the microwave system.

\section{EQUATIONS OF THE BRAGG CAVITY}

We consider a Bragg cavity, which represents a piece of a corrugated waveguide with a shift of the corrugation phase by $\pi$ in its middle (Fig. 1). Eigenmodes of such a cavity are formed as a superposition of two traveling counterpropagating partial waves (fast modes of a smooth-wall waveguide), which are coupled on the Bragg structure. Propagation of these waves and their mutual scattering on the corrugated cavity wall are described by
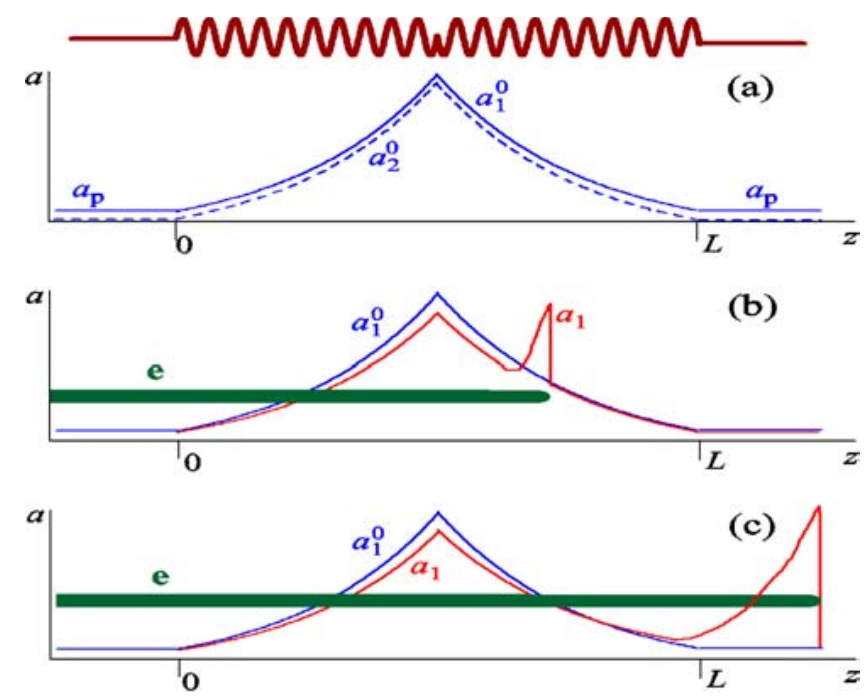

FIG. 1. (Color) Principle of generation of short $\mathrm{rf}$ pulses from a Bragg cavity pumped by an input rf signal. Microwave system and axial distribution of the cold (without electron beam) eigenmode, which is formed by forward $a_{1}^{0}(z)$ and backward $a_{2}^{0}(z)$ traveling waves coupled on the Bragg structure (a), as well as hot perturbations of the forward wave $a_{1}(t, z)$ in different moments of the time, when the front of the electron beam is still inside (b) and outside (c) the cavity. 
the following spatiotemporal equations $[12,13]$ :

$$
\begin{gathered}
\frac{1}{V} \frac{\partial a_{1}}{\partial t}+\frac{\partial a_{1}}{\partial z}=i \sigma a_{2} \phi(z)+F, \\
\frac{1}{V} \frac{\partial a_{2}}{\partial t}-\frac{\partial a_{2}}{\partial z}=i \sigma a_{1} \phi(z) .
\end{gathered}
$$

Here $t$ is the time, $z$ is the axial coordinate, $a_{1,2}$ are slow complex amplitude of the forward (index 1) and backward (index 2) partial waves, $V$ is the group velocity of the waves, and $\sigma$ is the factor of the Bragg coupling. The shift of the corrugation phase is described by the following function:

$$
\phi(z)= \begin{cases}1, & 0<z<L / 2 \\ -1, & L / 2<z<L\end{cases}
$$

where $L$ is the cavity length. In Eq. (1), function $F$ describes interaction of the electron beam with the forward wave $\left(a_{1}\right)$. It is assumed that the exact Bragg resonance takes place,

$$
h_{1}-h_{2}=2 \pi / d_{\text {cor }}
$$

where $h_{1,2}$ are the axial wave numbers of the waves, and $d_{\text {cor }}$ is the period of the corrugation. For instance, corrugation of the radius of a circular waveguide $R(z)=R_{0}+$ $R_{1} \phi(z) \cos \left(2 \pi z / d_{\text {cor }}\right)$ provides coupling between forward and backward waves with the same transverse structures with the following coupling factor:

$$
\sigma=\frac{R_{1}}{2 R_{0}^{2}} \frac{-\nu^{4}+R_{0}^{2}\left(\omega^{2} / c^{2}+h^{2}\right)}{h\left(\nu^{2}-1\right)}
$$

where $\quad \nu=R_{0} \sqrt{\omega^{2} / c^{2}-h^{2}} \quad$ (for the modes $\mathrm{TE}_{1,1}$ $\nu=1.84)$.

We consider the situation when the cavity is pumped with a long rf pulse, which enters the cavity input $(z=0)$. In this case the boundary conditions for the waves have the following form:

$$
a_{1}(0, t)=a_{\mathrm{p}}, \quad a_{2}(L, t)=0,
$$

where $a_{\mathrm{p}}$ is the amplitude of the pumping pulse.

Let us study the cold (without the electron beam, $F=$ $0)$ steady state, when $\partial a_{1,2} / \partial t=0$. The cold eigenmode $a_{1,2}^{0}(z)$ is expressed in the following form:

$$
\begin{aligned}
& a_{1}^{0}(z)=a_{\mathrm{p}} \cosh [\sigma(L / 2-|z-L / 2|)], \\
& a_{2}^{0}(z)=-i a_{\mathrm{p}} \sinh [\sigma(L / 2-|z-L / 2|)] .
\end{aligned}
$$

Let us note that the cold mode is characterized by the absence of a signal reflected from the cavity [Fig. 1(a)]:

$$
a_{2}^{0}(0)=0 \text {. }
$$

Both (forward and backward) traveling components of the cold eigen mode (4) have the maximum of their power in the center of the cavity, $z=L / 2$ [Fig. 1(a)]; for the forward component,

$$
P_{\max } / P_{\mathrm{p}}=\left|a_{1}^{0}(L / 2) / a_{\mathrm{p}}\right|^{2}=[\cosh (\sigma L / 2)]^{2} .
$$

Evidently, this value determines the cold quality factor of such a cavity: $Q \sim P_{\max } / P_{\mathrm{p}}$.

\section{FORMATION OF THE OUTPUT RF PULSE: MODEL AND ANALYSIS}

\section{A. Simplest model of the electron-wave interaction}

According to Eq. (5), if the cavity length is long enough, then the ratio between the stored power and the input signal power can be great even in the case of a weak Bragg coupling; only $\sigma L \gg 1$ is needed to provide a high $Q$ factor of the cavity. At the same time, weak wave coupling means that just a small perturbation of the axial wave number $\delta=h_{1}^{\text {hot }}-h_{1}$, namely, $\delta \sim \sigma$, results in a considerable perturbation of the cold eigenmode, which leads to radiation of the output $\mathrm{rf}$ pulse. Such perturbation of the axial wave number can be provided by a resonant interaction between the electron beam and the forward wave.

Let us consider the situation when the electron beam enters into the pumped cavity being in the cold steady state (Fig. 1), and study the transient process between the cold and the hot steady states. In order to introduce factor $F$ describing the interaction between electrons and the forward wave in Eq. (1), we use the following simplified model: we neglect the electron-wave energy exchange (which is possible if the electron beam power is much smaller than $P_{\max }$ ) and take into account only the influence of the electron-wave interaction on the axial wave number of the forward wave. In this model, factor $F$ is expressed in the following form:

$$
F=i \delta \cdot \eta\left(V_{\mathrm{e}} t-z\right) a_{1}, \quad \eta= \begin{cases}1, & V_{\mathrm{e}} t-z>0 \\ 0, & V_{\mathrm{e}} t-z<0,\end{cases}
$$

where $V_{\mathrm{e}}$ is the axial electron velocity and $\delta=h_{1}^{\text {hot }}-h_{1}$ is the difference between hot and cold axial wave numbers of the forward wave (the shift in the wave number caused by the electron-wave interaction). Heaviside function $\eta\left(V_{\mathrm{e}} t-z\right)$ corresponds to an ideally sharp front of the electron beam. The beam enters the cavity input, $z=$ 0 , at the moment $t=0$. Before this moment, the rf field distribution inside the cavity coincides with the cold eigenmode, Eq. (4):

$$
a_{1}(0, z)=a_{1}^{0}(z), \quad a_{2}(0, z)=a_{2}^{0}(z) .
$$

Certainly, the model used here is very artificial, it does not include some physical phenomena, and the electronwave interaction factor determined by Eq. (6) cannot be rigorously proven. However, the proposed model gives results, which are in very good accordance with numerical simulations of a system, where a significantly more realistic model of the electron-wave interaction is used (Sec. IV). 
For further analysis, it is convenient to introduce the hot perturbation of the complex amplitudes of the waves, $\tilde{a}_{1,2}=a_{1,2}-a_{1,2}^{0}$. Then Eqs. (1) and (2) are transformed to the following form:

$$
\begin{gathered}
\frac{1}{V} \frac{\partial \tilde{a}_{1}}{\partial t}+\frac{\partial \tilde{a}_{1}}{\partial z}=i \sigma \tilde{a}_{2} \phi(z)+F, \\
\frac{1}{V} \frac{\partial \tilde{a}_{2}}{\partial t}-\frac{\partial \tilde{a}_{2}}{\partial z}=i \sigma \tilde{a}_{1} \phi(z),
\end{gathered}
$$

with $F=i \delta \cdot \eta\left(V_{\mathrm{e}} t-z\right)\left(a_{1}^{0}+\tilde{a}_{1}\right)$ and the zero initial and boundary conditions

$$
\begin{aligned}
& \tilde{a}_{1}(t=0, z)=\tilde{a}_{2}(t=0, z)=0, \\
& \tilde{a}_{1}(t, z=0)=\tilde{a}_{2}(t, z=L)=0 .
\end{aligned}
$$

\section{B. Numerical simulations}

Let us consider the case when the axial electron velocity and the wave group velocity coincide, $V_{\mathrm{e}}=V$. In this case, Eqs. (8)-(10) can be normalized to Bragg coupling
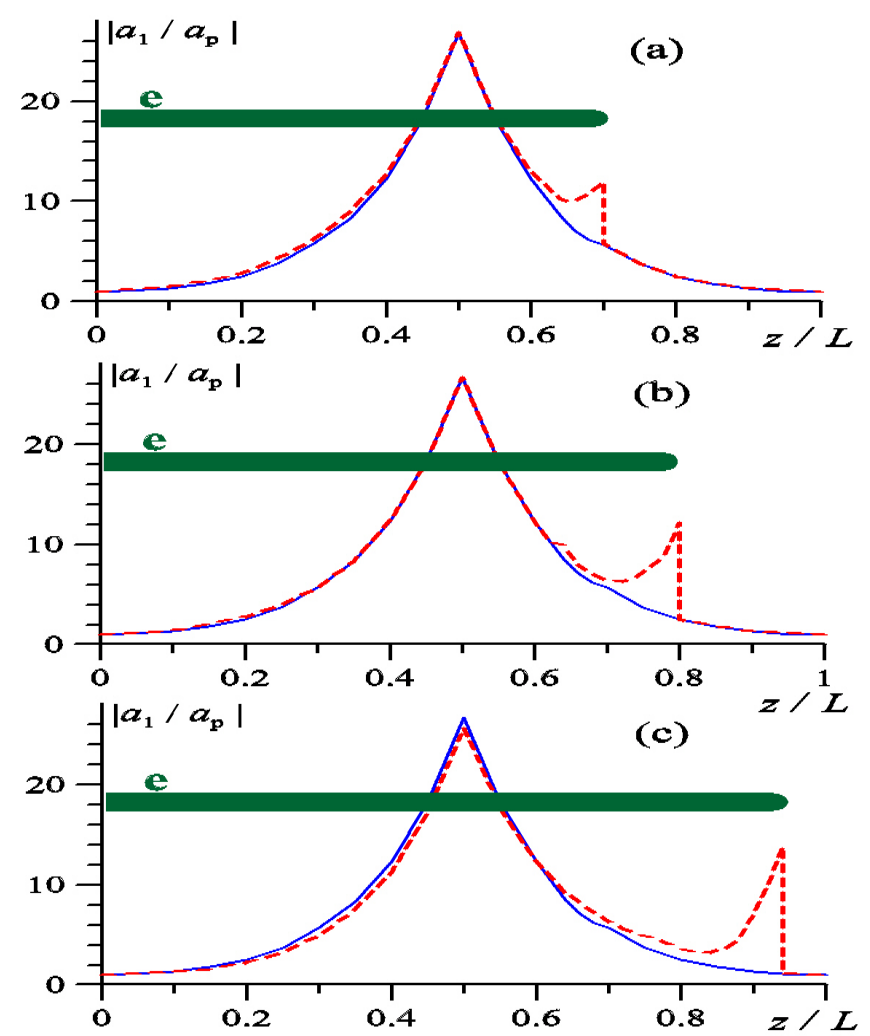

FIG. 2. (Color) Numerical simulations of the modeling system, the case of $\sigma L=8$ and $\delta / \sigma=0.3$. Pressing out of the output rf pulse by the electron beam. Hot perturbations of the field of the forward wave inside the cavity (red curves) on the background of the cold axial distribution of the forward wave (blue curves) in different moments of the time, $V t=0.70 L$ (a), $V t=0.80 L$ (b), and $V t=0.95 L$ (c). parameter $\sigma$ by introducing the following dimensionless variables: $t^{\prime}=\sigma V t, z^{\prime}=\sigma z$ and $\delta^{\prime}=\delta / \sigma$.

As an example, we study the case of $\sigma L=8$, when cold quality of the cavity is quite high, $a_{1}^{0}(L / 2) / a_{\mathrm{p}} \approx 27$ [Fig. 2(a)]. Figure 2 illustrates the process when the electron beam "presses out" the forward rf pulse from the cavity, which is preliminarily pumped by an input $\mathrm{rf}$ signal. Since $V_{\mathrm{e}}=V$, the front of the electron pulse coincides with the front of the hot perturbation of the forward wave. The maximal hot rf-wave perturbation occurs at the front of the electron beam. When the front passes through the cavity, the hot steady state just slightly differs from the cold eigenmode.

Figure 3 illustrates forward and backward output $\mathrm{rf}$ pulses, $a_{1}(t, z=L)$ and $a_{2}(t, z=0)$. The forward output pulse [Fig. 3(a)] has a sharp front and a quite short duration; it is significantly shorter than the duration of the whole transient process. While the hot perturbation of the axial wave number $\delta$ is small enough $(\delta<\sigma)$, the peak amplitude increases with the increase of $\delta$. The saturation occurs when $\delta \sim \sigma$, and the saturated peak amplitude of the output pulse is close to the cold rf amplitude at the center of the cavity, $a_{1}^{\text {peak }}(t) \approx$ $a_{1}^{0}(L / 2)$. At the same time, the duration of the output pulse is independent on $\delta$ and determined only by the

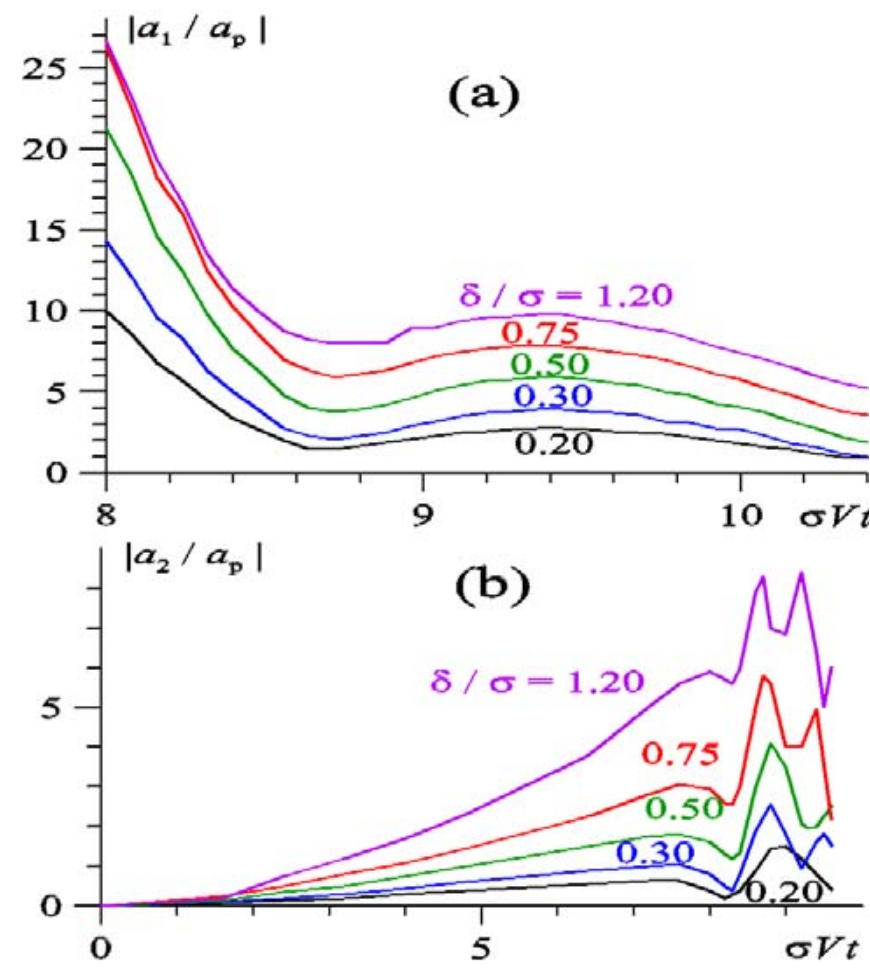

FIG. 3. (Color) Numerical simulations of the modeling system, the case of $\sigma L=8$. Forward (a) and backward (b) output rf pulses (wave amplitude versus the normalized time) at various values of the hot perturbation of the axial wave number $\delta$ normalized by the Bragg coupling factor $\sigma$. 
Bragg coupling parameter, $\sigma V T_{\text {pulse }} \sim 1$. Let us note that $T_{\text {pulse }}$ is significantly shorter than the duration of the transient process from the cold steady state to the hot one. As for the backward output pulse, it is quite long (its duration is of the order of the transient process), it has a smooth front, and its peak amplitude is relatively low [Fig. 3(b)].

According to simulation, the maximal peak amplitude of the output $\mathrm{rf}$ pulse is achieved when the condition of the "group synchronism" takes place, $V_{\mathrm{e}} \approx V$ (Fig. 4). Evidently the acceptable fault of this condition is determined by the requirement of a small enough divergence of the electron beam front and the front of hot forward-wave perturbation, $\left|L / V-L / V_{\mathrm{e}}\right|<T_{\text {pulse }}$, which leads to the following estimation:

$$
\frac{\left|V-V_{\mathrm{e}}\right|}{V}<\frac{1}{\sigma L}
$$

\section{Analysis of formation of the output rf pulse}

At the initial stage of the beam propagation inside the cavity, while $\sigma V t \ll 1$, one can neglect the Bragg wave coupling in Eq. (8). In addition, one can neglect the perturbation $\tilde{a}_{1}$ in $F$, assuming that it is small as compared with the initial field $a_{1}^{0}$. This approximation results in the following solution of Eqs. (8)-(10):

$$
\begin{gathered}
\tilde{a}_{1}(t, z)=i a_{\mathrm{p}} \frac{\delta}{\sigma} \eta(V t-z) \sinh (\sigma z), \\
\tilde{a}_{2}(t, z)=-a_{\mathrm{p}} \frac{\delta}{\sigma} \eta(V t-z)\left\{\cosh \left(\sigma \frac{z+V t}{2}\right)\right. \\
-\cosh (\sigma z)\} .
\end{gathered}
$$

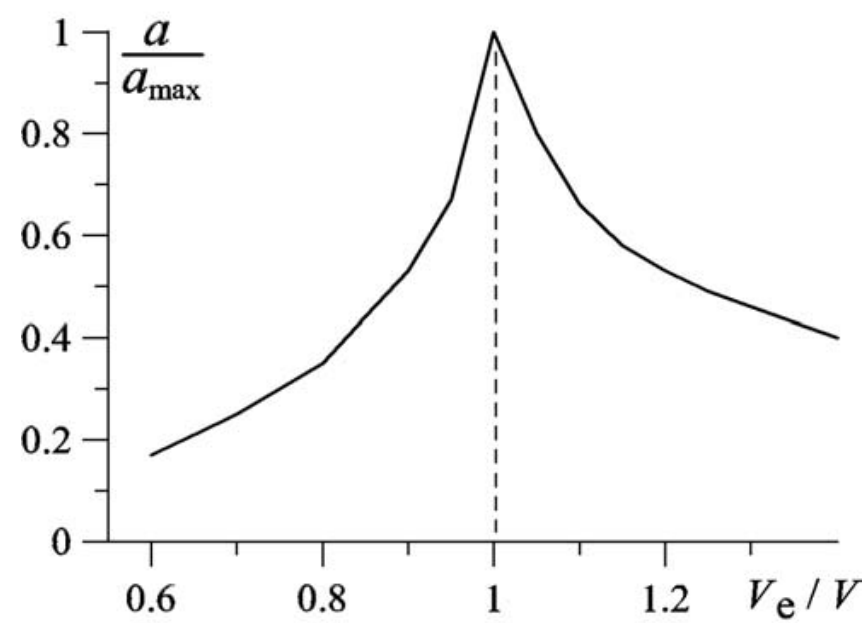

FIG. 4. Numerical simulations of the modeling system, the case of $\sigma L=8$. Peak amplitude of the forward $\mathrm{rf}$ pulse (normalized by its maximal value) versus the ratio of axial electron velocity and wave group velocity $V_{\mathrm{e}} / V$.
According to Eqs. (12) and (13), the front of the backward output rf pulse is smooth:

$$
\tilde{a}_{2}(t, z=0)=-a_{\mathrm{p}} \frac{\delta}{\sigma}[\cosh (\sigma V t / 2)-1]
$$

this confirms results of numerical simulations (Figs. 2 and 3). As for the forward-wave perturbation $\tilde{a}_{1}$, it has a sharp front, which propagates together with the electron beam front, $z=V t$, and increases in time. Thus, this "nondispersive" ( $\sigma V t \ll 1)$ approximation explains formation of the sharp front of the forward output rf pulse:

$$
\tilde{a}_{1}(t, z=L)=i a_{\mathrm{p}} \frac{\delta}{\sigma} \begin{cases}0, & t<L / V, \\ \sinh (\sigma V t), & t>L / V .\end{cases}
$$

However, this approximation does not explain mechanisms, which provide short duration of the output rf pulse, as well as saturation of its power.

Evidently the shortening of the forward output rf pulse occurs under the effect of the Bragg wave coupling on the pulse formation. This effect becomes important when $\sigma V t \sim 1$. This gives the following evident estimation for the length and the duration of the hot perturbation of the forward wave:

$$
L_{\text {pulse }} \sim 1 / \sigma, \quad T_{\text {pulse }} \sim 1 / \sigma V .
$$

The same result follows from an approximate solution of Eqs. (8)-(10), which can be obtained for sufficiently short times, $\sigma V t<L / 2$, using the Laplace transformation:

$$
\begin{aligned}
\tilde{a}_{1} \approx & -a_{\mathrm{p}} \exp (\sigma z)+a_{\mathrm{p}} \frac{\sigma}{\sigma-i \delta} \exp [\sigma z-(z-V t) \\
& \left.\times \frac{i \sigma \delta}{2(i \delta-\sigma)}\right] .
\end{aligned}
$$

Let us notice that the duration of the output pulse $T_{\text {pulse }}$ is determined only by the cold characteristics of the microwave system (namely, the group velocity of the forward wave $V$ and the Bragg coupling factor $\sigma$ ), and it is independent of the electron beam parameters. This is in good accordance with results of numerical simulations (Fig. 3).

Let us study the mechanism, which provides saturation of the peak power of the forward output rf pulse, which occurs at big enough values of the hot shift of the axial wave number $\delta$. According to the results of numerical simulations (Figs. 3 and 4 ) and the analysis given above, the power peak occurs in the front of the forward rf pulse, which coincides with the front of the electron pulse, $z=$ $V t$. At the same time, if $V_{\mathrm{e}}=V$, then Eqs. (8)-(10) have an analytical solution for the amplitude of the forward wave at the front of the electron pulse, $a_{1}(t=z / V$, $z)$. It can be obtained by means of substitution of the "old" variables $(t, z)$ with a new pair of variables $(\tau, z)$, where $\tau=V t-z$. Since at the front of the electron beam $\tau=0$, the backward-wave perturbation is absent $\tilde{a}(\tau=0, z)=$ 0 , the equation for the hot perturbation of the forward 
wave takes the following form:

$$
\frac{d \tilde{a}_{1}(\tau=0, z)}{d z}=i \delta\left[a_{1}^{0}(z)+\tilde{a}_{1}(\tau=0, z)\right] .
$$

This yields the following expression for the peak amplitude of the output rf pulse:

$$
\begin{aligned}
\tilde{a}_{1}^{\text {peak }}= & \tilde{a}_{1}(\tau=0, z=L) \\
= & i a_{\mathrm{p}} \delta\left[\frac{-2 i \delta \sin (\delta L / 2)+2 \sigma \sinh (\sigma L / 2)}{\delta^{2}+\sigma^{2}}\right] \\
& \times \exp (i \delta L / 2) .
\end{aligned}
$$

A high (as compared to the pumping signal $a_{\mathrm{p}}$ ) level of the output power is achieved when $\sigma L \gg 1$. In this case the expression for $\tilde{a}_{1}^{\text {peak }}$ is reduced to the following formula:

$$
\tilde{a}_{1}^{\text {peak }} \approx i a_{\mathrm{p}} \frac{2 \delta \sigma}{\delta^{2}+\sigma^{2}} \sinh (\sigma L / 2) \exp (i \delta L / 2) .
$$

According to Eq. (16), when hot shift of the axial wave number is small enough, $\delta<\sigma, \tilde{a}_{1}^{\text {peak }}$ is proportional to $\delta$ . Saturation occurs when $\delta$ becomes comparable with the Bragg coupling factor, $\delta \approx \sigma$, and the saturated peak amplitude is close to the cold $\mathrm{rf}$ amplitude in the center of the cavity, $\left|a_{1}^{\text {peak }}\right| \approx\left|a_{1}^{0}(L / 2)\right|$. This analytical prediction agrees with the numerical results (Fig. 3).

\section{CYCLOTRON-RESONANCE GENERATOR OF SHORT RF PULSES}

\section{A. Equations of the cyclotron generator}

In contrast to the previous section, where the electronwave interaction is introduced by means of a simple model, here we consider a more realistic case of a cyclotron-resonance generator. Let us consider the case when the pressing out of the output rf pulse is provided by a beam of electrons, which move inside the Bragg cavity along helical trajectories in the external homogeneous magnetic field, $\mathbf{z} B_{0}$. We suppose that at the input of the cavity the electrons have both axial and rotatory components of their velocity, $V_{\mathrm{e}}$ and $V_{\perp}$, and the following condition of cyclotron resonance between electrons and the forward wave takes place:

$$
\omega \approx h_{1} V_{\mathrm{e}}+\Omega,
$$

where $\Omega=e B_{0} / m c \gamma$ is the electron cyclotron frequency. In addition, we assume that the group synchronism condition $V_{\mathrm{e}} \approx V=c^{2} h_{1} / \omega$ is also satisfied.

We consider the case when the operating Bragg cavity represents a piece of a circular corrugated waveguide with a shift of the corrugation phase in its middle [Fig. 1(a)], and the electrons interact with the forward traveling wave $\mathrm{TE}_{1,1}$ at the fundamental cyclotron harmonic. Let us represent the relativistic Lorentz factor of an electron, $\gamma=1 / \sqrt{1-\left(V_{\mathrm{e}}^{2}+V_{\perp}^{2}\right) / c^{2}}$ in the form $\gamma=\gamma_{0}(W+1)$, and suppose that the electron-wave interaction results in a sufficiently small change in the electron energy, $W \ll 1$. In this case, equations of electron motion in the rf field of the resonant forward wave have the following form [14]:

$$
\begin{gathered}
\frac{d W}{d \zeta}=\chi \operatorname{Im} a_{1} e^{i \theta}, \\
\frac{d \theta}{d \zeta}=\left(\frac{\beta_{\mathrm{ph}}-b}{\beta_{\mathrm{e}}}-1\right)+\frac{\beta_{\mathrm{ph}} \beta_{\mathrm{e}}+\frac{b}{\beta_{\mathrm{ph}}}-1}{\beta_{\mathrm{e}}^{2}} W .
\end{gathered}
$$

Here $\zeta=h_{1} z$ is the normalized axial coordinate, $\theta=$ $\omega t-h_{1} z-\int \Omega d t$ is the electron phase with respect to the resonant wave, $\chi=\beta_{\perp} / \beta_{\mathrm{e}}$ is the electron-wave coupling factor, $\beta_{\perp, \mathrm{e}}=V_{\perp, \mathrm{e}} / c, \beta_{\mathrm{ph}}=\omega / c h_{1}$ is the phase velocity normalized to the speed of light, and $b=$ $e B_{0} / h_{1} m c^{2} \gamma_{0}$ is the normalized magnetic field. For the sake of simplicity, in Eq. (19) we have omitted the term describing the so-called "forced" electron bunching. The initial conditions for Eqs. (18) and (19) have the following form:

$$
W(0)=0, \quad \theta(0)=\varphi,
$$

where the initial electron phases $\varphi$ are distributed homogeneously over interval $[0,2 \pi)$.

In the case of the cyclotron-resonance electron-wave interaction, the interaction factor $F$ in Eq. (1) takes on the following form:

$$
F=i h_{1} G\left\langle e^{-i \theta}\right\rangle_{\varphi} \cdot \eta\left(V_{\mathrm{e}} t-z\right),
$$

where $G=I \frac{m c^{2}}{e} \frac{\gamma_{0} X}{2 N}$ is the excitation factor, $I$ is the electron current, $\langle\cdots\rangle_{\varphi}$ denotes averaging over all initial phases, and $N$ is the wave norm. Let us note that within the chosen normalization the power of the forward wave is related to the amplitude according to the following formula:

$$
P_{1}=N\left|a_{1}\right|^{2}
$$

\section{B. Required parameters of the system}

We suppose that the electron-wave resonance condition (17) is satisfied; correspondingly, $\beta_{\mathrm{ph}}-\beta_{\mathrm{e}}-b=0$. If the cavity length is long enough, then the electron phase shift is significant, $\Delta \theta \sim \pi$, and, therefore, one can assume that $\left\langle e^{-i \theta}\right\rangle_{\varphi} \sim 1$. According to Eq. (1), the electron beam significantly influences the propagation of the forward wave when $\sigma a_{2} \sim F$. Taking into account Eq. (4), one obtains the following estimation:

$$
G h_{1} \sim \sigma\left|a_{2}^{0}(L / 2)\right| .
$$

Since according to Eq. (16) in the saturated regime the peak amplitude of the output rf pulse is close to the rfwave amplitude in the cavity center, $\left|a_{1}^{\text {peak }}\right| \sim\left|a_{1,2}^{0}(L / 2)\right|$, 
one obtains

$$
G h_{1} \sim \sigma\left|a_{1}^{\text {peak }}\right|
$$

Let us consider two natural requirements, namely, smallness of both the electron beam power and the pumping $\mathrm{rf}$ power as compared to the peak power of the radiated rf pulse: $P_{1}^{\text {peak }} \gg P_{\mathrm{e}}$ [where $P_{\mathrm{e}}=\left(\gamma_{0}-\right.$ 1) $m c^{2} I / e$ is the electron beam power] and $a_{1}^{\text {peak }} \gg a_{\mathrm{p}}$. According to Eqs. (22) and (23), the first requirement is expressed as follows:

$$
\frac{N G^{2}}{P_{\mathrm{e}}} \frac{h_{1}^{2}}{\sigma^{2}} \gg 1 .
$$

According to Eq. (16), the second requirement is fulfilled if $\sigma L / 2 \gg 1$. Finally, one obtains

$$
\sqrt{\frac{P_{\mathrm{e}}}{N}} \frac{\gamma_{0} \chi}{\gamma_{0}-1} \frac{h_{1} L}{4}>\frac{\sigma L}{2} \gg 1 .
$$

\section{Numerical simulations}

In simulations we check the cyclotron-resonance generator of short rf pulses with a weakly relativistic electron beam. The main parameters of this system, as well as parameters of the output rf pulse predicted by simulations, are given in Table I. According to simulation, in this system the predicted peak power of the forward output rf pulse (8-11 MW) is significantly higher than both the input pumping rf power $(0.05 \mathrm{MW})$ and the electron beam power (3-4 MW), and of the order of the rf power circulating inside the cavity (36 MW).

Figures 5 and 6 illustrate the shape of the forward output rf pulses, as well as the temporal evolution of the hot axial structure of the rf field in the process of motion of the electron beam inside the cavity. Let us note that the behavior of the system studied here is very similar to the one of the modeling system (Figs. 2 and 3).

TABLE I. Parameters of the simulated cyclotron generator of short rf pulses.

\begin{tabular}{lc}
\hline \hline Frequency & $30 \mathrm{GHz}$ \\
Operating transverse mode & $\mathrm{TE}_{1,1}$ \\
Cavity radius & $0.34 \mathrm{~cm}$ \\
Cavity length & $80 \mathrm{~cm}$ \\
Electron energy & $100 \mathrm{keV}$ \\
Axial electron velocity & $0.530 c$ \\
Rotary electron velocity & $0.053 c$ \\
Electron current & $30 / 40 \mathrm{~A}$ \\
Electron beam power & $3 / 4 \mathrm{MW}$ \\
Pumping rf power & $0.05 \mathrm{MW}$ \\
rf power in the center of the cavity & $36 \mathrm{MW}$ \\
Peak power of the forward output rf pulse & $8 / 11 \mathrm{MW}$ \\
Duration of the output rf pulse & $0.4 \mathrm{ps}$ \\
\hline \hline
\end{tabular}

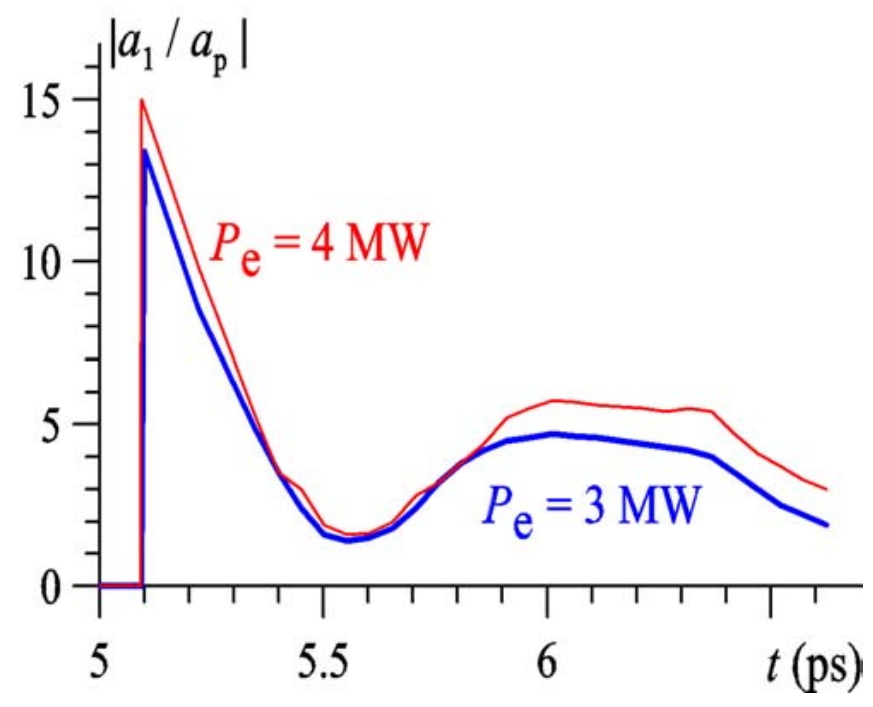

FIG. 5. (Color) Numerical simulations of the cyclotron generator. Forward output rf pulses (wave amplitude normalized to the pumping amplitude versus the time) at various values of the electron beam power.

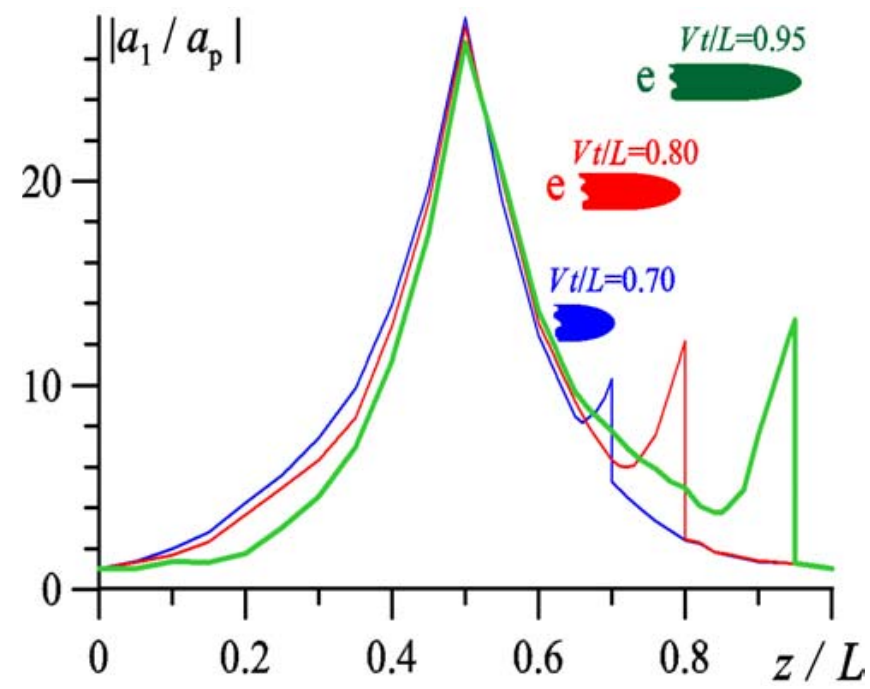

FIG. 6. (Color) Numerical simulations of the cyclotron generator, the case of $P_{\mathrm{e}}=3 \mathrm{MW}$. Pressing out of the output rf pulse by the electron beam. Hot axial structure of the forward rfwave field inside the cavity in different moments of time, $V t=$ $0.70 L$ (blue curve), $V t=0.80 L$ (red curve), and $V t=0.95 L$ (green curve).

\section{CONCLUSION}

In this work, a new method of generation of short powerful rf pulses is proposed. The method is based on the effect of pressing out of a short rf pulse by an electron beam from a Bragg cavity, which is pumped with a lowpower input rf signal. The electron beam slightly disturbs 
the cold (without the electron beam) steady state of the system, and the generation of the powerful output rf pulse occurs in the beginning of the transient process from the cold steady state to the hot one. This effect takes place if the group synchronism condition is fulfilled: the translational electron velocity is close to the group velocity of the wave circulating inside the cavity. The peak power of the output pulse is determined by the value of the electron current. It can be significantly higher than both the pumping rf power and the electron beam power, and it is limited by the value of the stored $\mathrm{rf}$ power (the power of the wave circulating inside the cavity). At the same time, the duration of the output pulse is independent of the electron beam characteristics and it is fixed by the cold characteristics of the microwave system (namely, by the parameter of the Bragg wave coupling).

One should notice that in the proposed method the energy of the output pulse is much lower than the rf energy stored inside the cavity. This means that the efficiency of the proposed generator operating in the single-pulse regime is very low. However, since the difference between the cold steady state and the hot one is very small, one steady state can be quickly transformed into another steady state. Evidently this can provide realization of the regime of sequence of short rf pulses, which possesses a significantly higher efficiency.

\section{ACKNOWLEDGMENTS}

This work is supported by the Russian Foundation for Basic Research, Project No. 02-02-17205, as well as by the Russian Science Support Foundation.
[1] R. A. Alvarez, Rev. Sci. Instrum. 57, 2481 (1986).

[2] M. I. Petelin, A. L.Vikharev, J. L. Hirshfield, in Advanced Accelerator Concepts: Seventh Workshop, edited by S. Chattopadhyay, J. McCullough, and P. Dahl, AIP Conf. Proc. No. 398 (AIP, New York, 1997), p. 822.

[3] S. G.Tantawi, in Advanced Accelerator Concepts: Eighth Workshop, edited by W. Laswon, AIP Conf. Proc. No. 472 (AIP, New York, 1999), p. 959.

[4] A. V.Savilov, Phys. Rev. Lett. 88, 064801 (2002).

[5] N. S. Ginzburg et al., Phys. Rev. Lett. 78, 2365 (1997).

[6] N. S.Ginzburg et al., Phys. Rev. E 60, 3297 (1999).

[7] D. M. Grishin, V. P. Gubanov, S. D. Korovin, S. K. Lyubutin, G. A. Mesyats, A.V. Nikiforov, V.V. Rostov, S. N. Rukin, B. G. Slovikovski, M. R. Ul'maskulov, K. A. Sharypov, V. G. Shpak, S. A. Shunalov, and M. I. Yalandin, Tech. Phys. Lett. 28, 806 (2002).

[8] A. A. El'chaninov, S. D. Korovin, V.V. Rostov, I.V. Pegel', G. A. Mesyats, M. I. Yalandin, and N. S. Ginzburg, JETP Lett. 77, 226 (2003).

[9] G. G. Denisov and M. G. Reznikov, Izv. Vyssh. Uchebn. Zaved., Radiofiz. 25, 562 (1982).

[10] V. L.Bratman, G. G. Denisov, N. S. Ginzburg, and M. I. Petelin, IEEE J. Quantum Electron. 19, 282 (1983).

[11] C. K. Chong, D. B. McDermott, M. M. Razeghi, N.C. Luhmann, Jr., J. Pretterebner, D. Wagner, M. Thumm, M. Caplan, and B. Kulke, IEEE Trans. Plasma Sci. 20, 393 (1992).

[12] V.Ya. Bogomolov, N.S. Ginzburg, and A.S. Sergeev, Radiotekh. Elektron. (Moscow) 31, 102 (1986) (in Russian).

[13] N. S. Ginzburg, N. Yu. Peskov, and A. S. Sergeev, Opt. Commun. 112, 151 (1994).

[14] V. L.Bratman et al., Int. J. Electron. 51, 541 (1981). 